\title{
Entscheidend ist der Einzelfall
}

\section{Die Schweigepflicht im beruflichen Alltag}

\section{INGRID SIEBRECHT}

Prof. Dr. Ingrid Siebrecht ist Hochschullehrerin für Soziale Arbeit und Bildung und Erziehung an der Hochschule für angewandte Wissenschaft und Kunst (HAWK) in Hildesheim. Ihre Arbeits- und Forschungsschwerpunkte sind Berufs- und Haftungsrecht, Zivilrecht und Recht der Grundsicherung für Arbeitsuchende.

ingrid.siebrecht@hawk-hhg.de

\author{
Staatlich anerkannte Sozialarbeiter und Sozialpädagogen \\ haben eine gesetzliche Schweigepflicht, die auch nicht \\ durch eine Weisung von Vorgesetzten aufgehoben \\ oder abgeschwächt werden kann. Doch in der \\ Praxis stellt sich Fachkräften oft die Frage, welche \\ Informationen sie an wen weitergeben dürfen.
}

Von Studierenden der Hochschule für angewandte Wissenschaft und Kunst in Hildesheim im Rahmen eines Forschungsprojekts durchgeführte anonyme Interviews haben gezeigt, dass häufig Unsicherheit über das Thema Schweigepflicht und seine Bedeutung im beruflichen Alltag von sozialen Einrichtungen herrscht. Häufiger wurde die Schweigepflicht als Risikofaktor (»immer mit einem Bein vor Gericht«) und Ballast (»umständlich«, "Zeitverlust «, »kostenintensiv«) empfunden. Einige Fragen tauchten in den Gesprächen wiederholt auf und sollen daher hier vertieft behandelt werden:

- Wie kann die kollegiale Zusammenarbeit innerhalb einer Einrichtung unter Berücksichtigung der Schweigepflicht ablaufen?

- Welche Grundsätze gelten für die Kooperation mit anderen Trägern, welche gegenüber Behörden und anderen Externen, beispielsweise Ärzten?

- Wie kann das Verhältnis zwischen den Mitarbeitenden der Einrichtung sowie den Angehörigen und Betreuern unter Berücksichtigung der Schweigepflicht ausgestaltet werden?

Entsprechend den aufgeworfenen Fragen wird im Folgenden unterschieden:

- Die Beachtung der Schweigepflicht der Beschäftigten einer Einrichtung untereinander, nämlich:

- zwischen den Mitarbeitern desselben Zuständigkeitsbereichs oder
- der Mitarbeiter gegenüber Verwaltung, Leitung anderen Abteilungen, und der

- Beachtung der Schweigepflicht gegenüber nicht in der Einrichtung Beschäftigten, nämlich

- Klienten, Angehörigen, Betreuern

- anderen externen Personen, z. B. Ärzten oder

- im dienstlichen Austausch mit sozialen Einrichtungen und Behörden.

\section{Grundsätzliches}

Wichtigstes und oberstes Gebot für die Beachtung der Schweigepflicht ist die Sparsamkeit bei der Weitergabe von Informationen. Weitergegeben werden soll so wenig wie möglich, aber so viel wie nötig, um den Zweck der Weitergabe zu erreichen.

Dieses ist ein Grundsatz, der im moderneren Datenschutzrecht ausdrücklich geregelt ist: das Gebot der Datensparsamkeit. Damit ist gemeint, dass nur die für den jeweiligen Zweck erforderlichen Daten erhoben und übermittelt werden sollen und die Erhebung und Übermittlung von nicht erforderlichen Daten grundsätzlich vermieden werden soll.

Übertragen auf den Bereich der Schweigepflicht würde dies bedeuten, dass jeder für sich eine gewisse Sorgfalt im Umgang mit Informationen einübt: Weshalb sammle ich bestimmte Informationen? Wie gehe ich mit gewonnenen Informationen um? $\mathrm{Zu}$ welchem Zweck gebe ich diese weiter? 


\section{Verletzung von Privatgeheimnissen}

(1) Wer unbefugt ein fremdes Geheimnis, namentlich ein zum persönlichen Lebensbereich gehörendes Geheimnis oder ein Betriebs- oder Geschäftsgeheimnis, offenbart, das ihm als

1. Arzt, Zahnarzt, Tierarzt, Apotheker oder Angehörigen eines anderen Heilberufs, der für die Berufsausübung oder die Führung der Berufsbezeichnung eine staatlich geregelte Ausbildung erfordert,

2. Berufspsychologen mit staatlich anerkannter wissenschaftlicher Abschlussprüfung,

3. Rechtsanwalt, Patentanwalt, Notar, Verteidiger in einem gesetzlich geordneten Verfahren, Wirtschaftsprüfer, vereidigtem Buchprüfer, Steuerberater, Steuerbevollmächtigten oder Organ oder Mitglied eines Organs einer Rechtsanwalts-, Patentanwalts-, Wirtschaftsprüfungs-, Buchprüfungs- oder Steuerberatungsgesellschaft,

4. Ehe-, Familien-, Erziehungs- oder Jugendberater sowie Berater für Suchtfragen in einer Beratungsstelle, die von einer Behörde oder Körperschaft, Anstalt oder Stiftung des öffentlichen Rechts anerkannt ist,

4a. Mitglied oder Beauftragten einer anerkannten Beratungsstelle nach den $\$ \S 3$ und 8 des Schwangerschaftskonfliktgesetzes,

5. staatlich anerkanntem Sozialarbeiter oder staatlich anerkanntem Sozialpädagogen oder

6. Angehörigen eines Unternehmens der privaten Kranken-, Unfall- oder Lebensversicherung oder einer privatärztlichen, steuerberaterlichen oder anwaltlichen Verrechnungsstelle

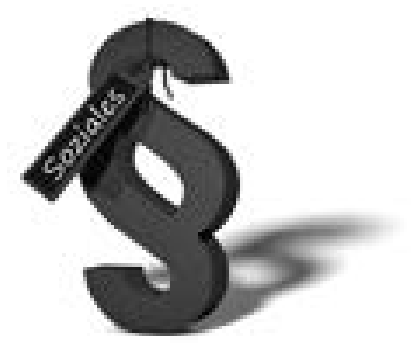

anvertraut worden oder sonst bekanntgeworden ist, wird mit Freiheitsstrafe bis zu einem Jahr oder mit Geldstrafe bestraft.

(2) Ebenso wird bestraft, wer unbefugt ein fremdes Geheimnis, namentlich ein zum persönlichen Lebensbereich gehörendes Geheimnis oder ein Betriebs- oder Geschäftsgeheimnis, offenbart, das ihm als

1. Amtsträger,

2. für den öffentlichen Dienst besonders Verpflichteten,

3. Person, die Aufgaben oder Befugnisse nach dem Personalvertretungsrecht wahrnimmt,

4. Mitglied eines für ein Gesetzgebungsorgan des Bundes oder eines Landes tätigen Untersuchungsausschusses, sonstigen Ausschusses oder Rates, das nicht selbst Mitglied des Gesetzgebungsorgans ist, oder als Hilfskraft eines solchen Ausschusses oder Rates,

5. öffentlich bestelltem Sachverständigen, der auf die gewissenhafte Erfüllung seiner Obliegenheiten auf Grund eines Gesetzes förmlich verpflichtet worden ist, oder

6. Person, die auf die gewissenhafte Erfüllung ihrer Geheimhaltungspflicht bei der Durchführung wissenschaftlicher Forschungsvorhaben auf Grund eines Gesetzes förmlich verpflichtet worden ist, anvertraut worden oder sonst bekanntgeworden ist. Einem Geheimnis im Sinne des Satzes 1 stehen Einzelangaben über persönliche oder sachliche Verhältnisse eines anderen gleich, die für Aufgaben der öffentlichen Verwaltung erfasst worden sind; Satz 1 ist jedoch nicht anzuwenden, soweit solche Einzelangaben anderen Behörden oder sonstigen Stellen für Aufgaben der öffentlichen Verwaltung bekanntgegeben werden und das Gesetz dies nicht untersagt.

(2a) Die Absätze 1 und 2 gelten entsprechend, wenn ein Beauftragter für den Datenschutz unbefugt ein fremdes Geheimnis im Sinne dieser Vorschriften offenbart, das einem in den Absätzen 1 und 2 Genannten in dessen beruflicher Eigenschaft anvertraut worden oder sonst bekannt geworden ist und von dem er bei der Erfüllung seiner Aufgaben als Beauftragter für den Datenschutz Kenntnis erlangt hat.

(3) Einem in Absatz 1 Nr. 3 genannten Rechtsanwalt stehen andere Mitglieder einer Rechtsanwaltskammer gleich. Den in Absatz 1 und Satz 1 Genannten stehen ihre berufsmäßig tätigen Gehilfen und die Personen gleich, die bei innen zur Vorbereitung auf den Beruf tätig sind. Den in Absatz 1 und den in Satz 1 und 2 Genannten steht nach dem Tod des zur Wahrung des Geheimnisses Verpflichteten ferner gleich, wer das Geheimnis von dem Verstorbenen oder aus dessen Nachlass erlangt hat.

(4) Die Absätze 1 bis 3 sind auch anzuwenden, wenn der Täter das fremde Geheimnis nach dem Tod des Betroffenen unbefugt offenbart.

(5) Handelt der Täter gegen Entgelt oder in der Absicht, sich oder einen anderen zu bereichern oder einen anderen zu schädigen, so ist die Strafe Freiheitsstrafe bis zu zwei Jahren oder Geldstrafe.

$\S 203$ des Strafgesetzbuches
Möglicherweise lässt sich schon durch diese einfachen Überlegungen unter Beachtung des Grundsatzes der Sparsamkeit mehr Handlungssicherheit im Umgang mit der Schweigepflicht erreichen.

Ein zweiter wichtiger Faktor im Umgang mit Informationen ist der Faktor Zeit. Häufig wird der Zusammenhang zur Schweigepflicht bei der Weitergabe von Informationen erst zu spät erkannt. In der Hektik des beruflichen Alltags erledigt man Anfragen beispielsweise schnell telefonisch, manchmal ohne die Vertraulichkeit zu bedenken. Sobald jedoch der Gedanke aufkommt, es könnte es sich um sensible Informationen handeln, über deren Weitergabe man noch nachdenken möchte, sollte man erst einmal schweigen. Eine Weitergabe von Informationen lässt sich nicht mehr rückgängig machen, eine kurzfristige Verzögerung der Weitergabe löst jedoch in der Regel keine schwerwiegenden Folgen aus.

Schließlich spielt auch die Möglichkeit zur Anonymisierung und zur Pseudonymisierung eine wichtige Rolle. Unter Anonymisierung versteht man die Weitergabe von Informationen ohne dass ein Bezug zu der betroffenen Person hergestellt werden kann. Unter Pseudonymisierung die Schilderung eines entsprechenden Sachverhalts unter Verwendung eines fremden Namens für die betroffene Person. Beide Möglichkeiten können jedoch nur dann greifen, wenn es nicht gerade wichtig ist, den Bezug zu dem betroffenen Klienten herzustellen. Ihr Anwendungsbereich bleibt daher beschränkt.

\section{Die Beachtung der Schweigepflicht innerhalb einer Einrichtung}

Innerhalb einer Organisation müssen bei der Datenweitergabe vor allem zwei Fälle berücksichtigt werden: gegenüber anderen Mitarbeitern in der Einrichtung und gegenüber anderen Abteilungen und an Vorgesetzte. 


\section{Gegenüber anderen Mitarbeitern der Einrichtung}

Die Weitergabe von Informationen betreffend einzelne Klienten unter Kollegen desselben Zuständigkeitsbereichs ist regelmäßig durch eine - meist stillschweigende - Einwilligung der Angehörigen oder Betreuer abgesichert. Zum einen besteht häufig die Möglichkeit, bereits im Rahmen des Aufnahmevertrags mit der Einrichtung eine entsprechende Einverständniserklärung einzuholen. Zum anderen wissen die Angehörigen und Betreuer, dass ein kollegialer Austausch stattfinden muss, um eine optimale Betreuung zu gewährleisten. Nur wenn Klienten oder Angehörige und Betreuer ausdrücklich um Vertraulichkeit bitten, ist Vorsicht geboten. Auch in diesen Fällen kann in einer Notstandssituation eine Weitergabe erlaubt sein, um eine drohende Gefahr abzuwenden. Es sollte jedoch immer vorher eine Abwägung vorgenommen und das Vorgehen mit fachlichen Vorgesetzten unter Umständen unter Einholung juristischer Beratung abgesprochen werden. Die entscheidenden Überlegungen für die Abwägung sind:

- $\mathrm{Zu}$ welchem Zweck möchte ich die Information weitergeben?

- Wie wird die Situation sein, wenn ich schweige?

Helfen kann dabei folgende Abwägung: Ist die Einhaltung der Schweigepflicht wichtiger oder ein Eingreifen unter Weitergabe der anvertrauten Informationen?

\section{Weitergabe an Vorgesetzte, die Verwaltung oder andere Abteilungen}

Die erläuterten Grundsätze gelten nicht nur für die kollegiale Zusammenarbeit, sondern auch für die Zusammenarbeit mit Vorgesetzten im selben Zuständigkeitsbereich. Wie ist es aber, wenn beispielsweise Mitarbeiter der Personalverwaltung, anderer Abteilungen oder der Vorstand einer Einrichtung detaillierte Informationen über einzelne Klienten verlangen?

Im Zweifel ist ein Klient und seine Angehörigen und Betreuer nur mit der Weitergabe zu einem bestimmten Zweck, zum Beispiel der Betreuung in der Einrichtung, einverstanden. Werden also beispielsweise Daten zur Hygieneversorgung oder zum Medikamentenbedarf abgefragt, wäre dies vom Zweck »Betreuung in der Einrichtung " gedeckt und der Mitarbeiter dürfte die Auskünfte erteilen. Sollen die Informationen über den Klienten jedoch beispielswei-
Bei Kindern, also bis zum Alter von 14 Jahren, gilt: Der Austausch von vertraulichen Informationen erfolgt in der Regel mit den Sorgeberechtigten. So lange ein Mitarbeiter nicht weiß, dass beispielsweise im Rahmen einer Ehescheidung einem Elternteil das Sorge-

"Grundsätzlich gilt das Gebot der 'Datensparsamkeit' auch in der

\section{Sozialen Arbeit»}

se an Außenstehende zu Werbe- oder geschäftlichen Zwecken weitergegeben werden, wäre hierfür von der anfragenden Abteilung eine ausdrückliche Einverständniserklärung einzuholen oder die Daten sollten anonymisiert oder pseudonymisiert weitergegeben werden.

\section{Die Beachtung der Schweigepflicht gegenüber Externen}

Bei der Weitergabe von vertraulichen Informationen nach außen ist besondere Vorsicht geboten.

\section{Weitergabe an Klienten, Angehörige, Betreuer}

Die Weitergabe von Informationen über einzelne Klienten an andere Klienten oder an deren Angehörige und Betreuer oder auch nur das Mithörenlassen Unbeteiligter eines beispielsweise im Flur geführten vertraulichen Gesprächs kann leicht zu einer Verletzung der Schweigepflicht führen.

Mitarbeiter sollten sich dessen bewusst sein und Gelegenheiten für vertrauliche Situationen schaffen, was in turbulenten Situationen, beispielsweise Abholzeiten, Schwierigkeiten bereiten kann. Auch hier gilt die oben formulierte Abwägungsregel sinngemäß:

- Zu welchem Zweck möchte ich die Information weitergeben?

- Wie wird die Situation sein, wenn ich schweige?

Helfen kann auch dabei die folgende Abwägung: Ist die Einhaltung der Schweigepflicht wichtiger oder ein Eingreifen unter Weitergabe der anvertrauten Informationen? recht nicht mehr zusteht, darf er vom Regelfall des gemeinsamen Sorgerechts ausgehen.

Sorgeberechtigte dürfen weitere Personen, beispielsweise Großeltern, ermächtigen, Informationen weiterzugeben und zu empfangen. Diese Ermächtigung sollte zur Sicherheit schriftlich festgehalten werden.

Wenn ein Kind dem Mitarbeiter einer Einrichtung Informationen anvertraut, die seine Sorgeberechtigten unmittelbar betreffen, beispielsweise über die Anwendung körperlicher Gewalt am Kind durch die Eltern, ist der Mitarbeiter nicht verpflichtet, die Information an die Sorgeberechtigten weiterzugeben. Hier wäre zum Schutz des Kindes ein Austausch mit weiteren Fachkräften und gegebenenfalls die Hinzuziehung des Jugendamtes geboten.

Jugendliche, also ab 14 Jahre, genießen selbst Vertrauensschutz. Dieser steht allerdings bis zum Erreichen der Volljährigkeit neben dem oben beschriebenen Sorgerecht der Eltern. Mitarbeiter müssen hier im Einzelfall entscheiden: Welche Informationen des Jugendlichen gebe ich an die Sorgeberechtigten weiter und umgekehrt? Aus rechtlicher, nicht aus pädagogischer Sicht ist diese Entscheidung unproblematisch, denn die Weitergabe von Informationen erfolgt im Verhältnis Jugendlicher und Eltern niemals an unbefugte Dritte, da beide informationsberechtigt sind. Damit scheidet eine Verletzung der rechtlichen Schweigepflicht aus.

Bei volljährigen Klienten kommt es auf die natürliche Einsichts- und Urteilsfähigkeit an. Alte oder geistig behinderte Menschen haben, auch wenn sie unter rechtlicher Betreuung stehen, grundsätzlich ebenso ein Recht auf 
Vertraulichkeit wie alle Volljährigen. Hinsichtlich der Einwilligung in die Weitergabe von vertraulichen Informationen müssen diese Menschen in der Lage sein, die Bedeutung ihrer Einwilligung zu erkennen und sie müssen einen zustimmenden Willen zum Ausdruck bringen können. Sollte dies nicht möglich sein, nimmt der Betreuer die Einwilligung für den Klienten nach den beschriebenen Grundsätzen vor. Vertrauliche Informationen sind dann mit ihm anstelle des Klienten auszutauschen.

\section{Weitergabe von Informationen an Externe}

Eine Weitergabe von Informationen, die der Schweigepflicht unterliegen, ist, wie erläutert, nicht schon deshalb zulässig, weil der Empfänger der Information selbst der Schweigepflicht unterliegt. Vielmehr kommt es auch hier in erster Linie auf den Willen und das (stillschweigende) Einverständnis des Klienten und seiner Angehörigen und Betreuer mit der Weitergabe an. Liegt ein solches vor, ist die Informationsweitergabe unproblematisch.

Ohne das Einverständnis ist entsprechend den datenschutzrechtlichen Grundsätzen auf den Zweck der Informationsweitergabe abzustellen. Erfolgt sie, um eine Gefahr vom Klienten abzuwenden, die schwerer wiegt als die Einhaltung der Schweigepflicht, ist sie nach den Grundsätzen des rechtfertigenden Notstands zulässig.

\section{Weitergabe von Informationen an andere soziale Einrichtungen oder Behörden}

Bei der Weitergabe von Informationen an andere soziale Einrichtungen ist der Zweck der Informationsweitergabe zu beachten. Geht es um die Unterstützung bei oder die Übernahme der Betreuung eines Klienten, ist Vorsicht geboten. Sofern es sich um eine übliche Maßnahme handelt, wird der Klient bereits zu Beginn der Zusammenarbeit darüber informiert worden und damit einverstanden sein. Anderenfalls müsste das Einverständnis des Klienten in die Weitergabe von der Schweigepflicht unterliegenden Informationen an die andere soziale Einrichtung eingeholt werden. Hieran sind keine besonderen Anforderungen, wie etwa Schriftlichkeit geknüpft. Ein zwanglo- ses Gespräch, über das eine Aktennotiz gemacht wird, reicht in diesem Fall aus. Auch eine unter dem Gesichtspunkt der rechtfertigenden Notstands erlaubte Weitergabe von Informationen an eine andere Einrichtung kommt in Betracht, wenn es um höherrangige Interessen des Klienten geht.

Die Weitergabe von Informationen an das Jugendamt ist zur Abwehr einer Kindeswohlgefährdung gesetzlich vorgeschrieben; ebenso die Weitergabe von Informationen über bestimmte hoch ansteckende Krankheiten an die Gesundheitsbehörden.

Gegenüber der Polizei müssen Mitarbeiter sozialer Einrichtungen, unabhängig davon, ob es sich um solche in privater oder in staatlicher Trägerschaft handelt, nur bestimmte Informationen betreffend Name, Aufenthaltsort oder Arbeitsstelle weitergeben.

Wurde gegen einen Klienten ein Strafverfahren eröffnet, sind Mitarbeiter hingegen zur Weitergabe von allen Informationen verpflichtet, die zur Durchführung des Verfahrens erforderlich sind. Klientenakten dürfen aufgrund eines entsprechenden richterlichen Beschlusses sogar beschlagnahmt werden.

\section{Schlussbetrachtung}

Eine Interessenabwägung im Einzelfall bleibt Mitarbeitern nicht erspart. Aber: Die Überprüfung, ob ein Mitarbeiter eine Verletzung der ihm obliegenden Schweigepflicht begangen hat, wird letztlich von einem Gericht vorgenommen. Dabei kommt es nicht darauf an, ob der Mitarbeiter als juristischer Laie bei der Interessenabwägung zu demselben Ergebnis gelangt ist, zu dem auch das Gericht kommt.

Entscheidend für die nachträgliche juristische Bewertung des Verhaltens ist die Frage, ob der Mitarbeiter sich ausreichend informiert und »sein Gewissen angespannt « hat. Je ungewöhnlicher und komplizierter der konkrete Fall sich für den Mitarbeiter darstellt, um so eher sollte er fachkundigen Rat in Anspruch nehmen. Einem Mitarbeiter jedoch, der in einer einfachen Standardsituation auf der Basis seines (möglichst aktuellen) Fachwissens eine »falsche« Abwägung vornimmt, fehlt das Bewusstsein, sich strafbar zu machen. Eine Verletzung der Schweigepflicht könnte ihm nicht vorgeworfen werden.
Sofern Mitarbeiter sich also mit dem Thema berufliche Schweigepflicht vertraut gemacht und ihr Wissen hin und wieder aufgefrischt haben, darf jeder zumindest in alltäglichen, einfach gelagerten Situationen auf seine Fachkompetenz vertrauen. Das Gefühl, mit einem Bein vor Gericht zu stehen, müsste der Handlungssicherheit weichen.

\section{Literatur}

Kliemann, Fegert: Informationsweitergabe im Kinderschutz, ZRP 2011, $110 \mathrm{ff}$.

Wilmertz, Rauschert: Datenschutz in der freien Jugend- und Sozialhilfe, Stuttgart 2004. Paul: Auswirkungen des $\S 8$ a auf Schweigepflicht und Sozialdatenschutz, Forum für Kinder- und Jugendarbeit 2008, 32 ff. Pollähne, Rohde: Schweigepflicht und Datenschutz, Berlin 2010.

\section{JAHRE Diakonie $\mathrm{m}$ Katastrophenhilfe}

\section{Weltweit hilfsbereit.}

Soforthilfe, Wiederaufbau und Prävention. Jeden Tag. Weltweit.

Ihre Spende hilft.

IBAN: DE26 210602370000502502 